CARPATHIAN J. MATH.

Volume 38 (2022), No. 2,

Pages 405 - 415

\title{
Monotone iteration method for general nonlinear two point boundary value problems with deviating arguments
}

\author{
Bapurao C. Dhage ${ }^{1}$, Janhavi B. Dhage ${ }^{1}$ and Javid Ali ${ }^{2}$
}

\begin{abstract}
In this paper we shall study the existence and approximation results for a nonlinear two point boundary value problem of a second order ordinary differential equation with general form of Dirichlet/Neumann type boundary conditions. The nonlinearity present on the right hand side of the differential equation is assumed to be Carathoéodory containing a deviating argument. The proofs of the main results are based on a monotone iteration method contained in the hybrid fixed point principles of Dhage (2014) in an ordered Banach space. Finally, some remarks concerning the merits of our monotone iteration method over other frequently used iteration methods in the theory of nonlinear differential equations are given in the conclusion.
\end{abstract}

\section{INTRODUCTION}

Let $\mathbb{R}$ denote the set of all real numbers and $\mathbb{R}_{+}$the set of all nonnegative reals. Given a closed and bounded interval $I=\left[t_{0}, t_{1}\right] \subset \mathbb{R}, t_{0}<t_{1}$, consider the nonlinear two point BVP of ordinary second order differential equation with a deviating argument

$$
\mathcal{L} x(t)=f(t, x(t), x(\varphi(t))) \text { a.e. } t \in I,
$$

where operator $\mathcal{L}: A C^{1}(I) \rightarrow L^{1}(I)$ has the form

$$
\mathcal{L} x=-x^{\prime \prime}+p(t) x^{\prime}+q(t) x,
$$

where $p, q \in L^{\infty}(I)$ and $q$ is positive-valued, $f: I \times \mathbb{R} \times \mathbb{R} \rightarrow \mathbb{R}$ and $\varphi$ is a continuous real function on $I$. Choosing an interval $J=\left[s_{0}, s_{1}\right]$ which contains both $I$ and $\varphi[I]$, and denoting $I_{0}=\left[s_{0}, t_{0}\right]$ and $I_{1}=\left[t_{1}, s_{1}\right]$. We shall adjoin to the differential equation (1.1) the following boundary condition:

$$
\mathcal{B}_{j} x(t)=a_{j} x(t)-(-1)^{j} b_{j} x^{\prime}(t)=\alpha_{j}(t), t \in I_{j}, j=0,1,
$$

where

$$
a_{j}, b_{j} \in \mathbb{R}_{+}, a_{j}+b_{j}>0 \text { and } \alpha_{j} \in L^{1}\left(I_{j}\right), j=0,1 .
$$

Definition 1.1. A function $x \in A C^{1}(J, \mathbb{R})$ is said to be a lower solution of the BVP (1.1) and (1.2) if

$$
\left.\begin{array}{r}
\mathcal{L} x(t) \leq f(t, x(t), x(\varphi(t))) \quad \text { a.e. } \quad t \in I, \\
\mathcal{B}_{j} x(t) \leq \alpha_{j}(t), t \in I_{j}, j=0,1,
\end{array}\right\}
$$

where $A C^{1}(J, \mathbb{R})$ is the space of functions $x \in C(J, \mathbb{R})$ whose first derivative exists and is absolutely continuous on $J$. Similarly, $x \in A C^{1}(J, \mathbb{R})$ is called an upper solution of (1.1) and (1.2) on $J$ if the reversed inequalities hold in (1.3). If equalities hold in (1.3), we say that $x$ is a solution of (1.1) and (1.2) on $J$.

Received: 16.06.2021. In revised form: 19.11.2021. Accepted: 26.11.2021

2010 Mathematics Subject Classification. 34A45, 34B15, 47H07, 47H10.

Key words and phrases. Nonlinear two point boundary value problem, deviating arguments, monotone iteration method, Existence and approximation theorem.

Corresponding author: Javid Ali; javid.mm@amu.ac.in 
In the case when $f$ is zero function, the problem (1.1) and (1.2) has a unique solution which we denote by $z$. In the special case when $f$ is a function of $t$ alone, it can be shown that the problem (1.1) and (1.2) has at most one solution in the class $A C^{1}(J, \mathbb{R})$. The existence of the solution is guaranteed by the above regularity and boundary conditions, and can be extended via boundary conditions to a unique solution of (1.1) and (1.2) on $J$.

The existence and uniqueness of the solution to the problem (1.1) and (1.2) is discussed in $[6,7]$ by using classical comparison and iteration methods, and assuming that $f$ is a continuous function in all its three arguments. The existence of extremal solutions to the BVP (1.1) and (1.2) between the given lower and upper solutions is studied in [8] via generalized iteration method, but allowing discontinuous nonlinearity for $f$. In the present paper, we shall study the existence and approximation of solutions of the differential problem (1.1) and (1.2) without usual Lipschitz condition as well as without assuming the existence of both comparable lower and upper solutions but via a monotone iteration method developed in Dhage [1, 2].

\section{Auxiliary Results}

We shall impose certain natural conditions on the nonlinear function $f: I \times \mathbb{R} \times \mathbb{R} \rightarrow \mathbb{R}$ in our discussion. So, we need the following useful definitions in the sequel.

Definition 2.2. $[1,2,3,4]$ An upper-semicontinuous and monotone nondecreasing function $\psi: \mathbb{R}_{+} \rightarrow \mathbb{R}_{+}$satisfying the condition $\psi(0)=0$ is called a $\mathcal{D}$-function on $\mathbb{R}_{+}$. The class of all $\mathcal{D}$-functions is denoted by $\mathfrak{D}$.

A few examples of the $\mathcal{D}$ functions on $\mathbb{R}_{+}$along with some applications appear in Dhage and Dhage [5] and references therein.

Definition 2.3. A function $\beta: I \times \mathbb{R} \times \mathbb{R} \rightarrow \mathbb{R}$ is called Carathéodory if

(i) the map $t \mapsto \beta(t, x, y)$ is measurable for each $x, y \in \mathbb{R}$, and

(ii) the map $(x, y) \mapsto \beta(t, x, y)$ is jointly continuous for each $t \in I$.

The following lemma is often used in the study of nonlinear differential equations (see Dhage and Heikkillä [8] and references therein).

Lemma 2.1 (Carathéodory). Let $\beta: I \times \mathbb{R} \times \mathbb{R} \rightarrow \mathbb{R}$ be a Carathéodory function. Then the map $(t, x, y) \mapsto \beta(t, x, y)$ is jointly measurable. In particular the map $t \mapsto \beta(t, x(t), y(t))$ is measurable on $I$ for all $x, y \in C(I, \mathbb{R})$.

We need the following hypotheses in the sequel.

$\left(\mathrm{H}_{1}\right)$ The function $f$ is Carathéodory on $I \times \mathbb{R} \times \mathbb{R}$.

$\left(\mathrm{H}_{2}\right) \quad f$ is bounded on $I \times \mathbb{R} \times \mathbb{R}$ with bound $M_{f}$.

$\left(\mathrm{H}_{3}\right) f(t, x, y)$ is nondecreasing in $x$ and $y$ for each $t \in I$.

$\left(\mathrm{H}_{4}\right)$ There exists a $\mathcal{D}$-function $\psi_{f} \in \mathfrak{D}$ such that

$$
0 \leq f\left(t, x_{1}, x_{2}\right)-f\left(t, y_{1}, y_{2}\right) \leq \psi_{f}\left(\max \left\{x_{1}-y_{1}, x_{2}-y_{2}\right\}\right)
$$

for all $\in I$ and $x_{1}, x_{2}, y_{1}, y_{2} \in \mathbb{R}$ with $x_{1} \geq y_{1}, x_{2} \geq y_{2}$.

(LS) The BVP (1.1) and (1.2) has a lower solution $u \in A C^{1}(J, \mathbb{R})$.

(US) The BVP (1.1) and (1.2) has an upper solution $v \in A C^{1}(J, \mathbb{R})$.

Lemma 2.2. Given any function $h \in L^{1}(I, \mathbb{R})$, the BVP

$$
\left.\begin{array}{c}
\mathcal{L} x(t)=h(t) \quad \text { a.e. } \quad t \in I, \\
\mathcal{B}_{j} x(t)=0, t \in I_{j}, j=0,1,
\end{array}\right\}
$$


is equivalent to the integral equation

$$
x(t)=\int_{t_{0}}^{t_{1}} k(t, s) h(s) d s, t \in I,
$$

where $k(t, s)$ is the Green's function associated with the homogeneous boundary value problem

$$
\left.\begin{array}{c}
-x^{\prime \prime}(t)+p(t) x^{\prime}(t)+q(t) x(t)=0 \text { a.e. } t \in I, \\
a_{j} x\left(t_{j}\right)-(-1)^{j} b_{j} x\left(t_{j}\right)=0, j=0,1 .
\end{array}\right\}
$$

Notice that the function $x$ given by (2.5) belongs to the class $C(I, \mathbb{R})$. Since $q \in C_{+}(I, \mathbb{R})$, it follows from the maximum principle that $k$ is continuous and nonnegative on $I \times I$. Therefore, the number $K=\sup _{t, s \in I} k(t, s)$ exists.

Employing the Green's function for ordinary linear differential equation, one can prove the following result.

Lemma 2.3. Suppose that the assumptions $\left(H_{1}\right)$ and $\left(H_{2}\right)$ hold. Then the BVP (1.1) and (1.2) can be expressed into the equivalent functional equation as

$$
x(t)=z(t)+ \begin{cases}\mathcal{F}_{f} x(t), & t \in I, \\ e^{\left(\frac{a_{j}\left(t-t_{0}\right)}{b_{j}}\right)} \mathcal{F}_{f} x\left(t_{j}\right), & t \in I_{j}, b_{j} \neq 0, j=0,1, \\ 0, & t \in I_{j}, b_{j}=0, j=0,1,\end{cases}
$$

where $z \in C(J, \mathbb{R})$ is a unique solution of the linear BVP

$$
\left.\begin{array}{l}
-x^{\prime \prime}(t)+p(t) x^{\prime}(t)+q(t) x(t)=0 \text { a.e. } t \in I, \\
a_{j} x\left(t_{j}\right)-(-1)^{j} b_{j} x\left(t_{j}\right)=\alpha_{j}(t), t \in I_{j}, j=0,1,
\end{array}\right\}
$$

and $\mathcal{F}_{f}$ is a continuous superposition operator on $C(I, \mathbb{R})$ defined by

$$
\mathcal{F}_{f} x(t)=\int_{t_{0}}^{t_{1}} k(t, s) f(s, x(s), x(\varphi(s))) d s, t \in I .
$$

The proof of our main results will be based on the monotone iteration principle or monotone iteration method embodied in the following applicable hybrid fixed point theorems of Dhage [1,2] in a partially ordered normed linear space.

Theorem $2.1([1,2])$. Let $(E, \preceq,\|\cdot\|)$ be a regular partially ordered complete normed linear space and let every compact chain $C$ in $E$ be Janhavi set. Let $\mathcal{T}: E \rightarrow E$ be a monotone nondecreasing, partially continuous and partially compact operator. If there exists an element $x_{0} \in E$ such that $x_{0} \preceq \mathcal{T} x_{0}$ or $x_{0} \succeq \mathcal{T} x_{0}$, then the hybrid operator equation $\mathcal{T} x=x$ has a solution $x^{*}$ in $E$ and the sequence $\left\{\mathcal{T}^{n} x_{0}\right\}_{n=0}^{\infty}$ of successive iterations converges monotonically to $x^{*}$.

Theorem $2.2([1,2])$. Let $(E, \preceq,\|\cdot\|)$ be a partially ordered Banach space and let $\mathcal{T}: E \rightarrow E$ be a monotone nondecreasing and nonlinear partial $\mathcal{D}$-contraction. Suppose that there exists an element $x_{0} \in E$ such that $x_{0} \preceq \mathcal{T} x_{0}$ or $x_{0} \succeq \mathcal{T} x_{0}$. If $\mathcal{T}$ is continuous or $E$ is regular, then $\mathcal{T}$ has a unique comparable fixed point $x^{*}$ and the sequence $\left\{\mathcal{T}^{n} x_{0}\right\}_{n=0}^{\infty}$ of successive iterations converges monotonically to $x^{*}$. Moreover, the fixed point $x^{*}$ is unique if every pair of elements in $E$ has a lower bound or an upper bound.

The details of monotone iteration principle or method and related definitions such as Janhavi set, Janhavi Banach space, partial continuity, partial compactness, partial $\mathcal{D}$ contraction and comparable fixed point of the operator etc., along with some nice applications may be found in Dhage [1, 2, 3, 4], Dhage and Dhage [6], Dhage et al. [7] and the references therein. 


\section{EXISTENCE AND APPROXIMATION RESULTS}

Let $C_{+}(J, \mathbb{R})$ denote the space of all nonnegative-valued functions of $C(J, \mathbb{R})$. We assume that the space $C(J, \mathbb{R})$ is endowed with the norm $\|\cdot\|$ and with the partial ordering $\preceq$ defined by

$$
\|x\|=\max _{t \in J}|x(t)|
$$

and

$$
x \preceq y \Longleftrightarrow x(t) \leq y(t) \forall t \in J .
$$

Clearly, $C(J, \mathbb{R})$ is a Banach space with respect to above maximum norm and also partially ordered with respect to the above partially order relation $\preceq$. It is known that the partially ordered Banach space $C(J, \mathbb{R})$ is regular and lattice so that every pair of elements of $C(J, \mathbb{R})$ has a lower and an upper bound in it (for details, see Dhage [1]- [3] and the references therein). The following useful lemma concerning the Janhavi subsets of $C(J, \mathbb{R})$ follows immediately from the Arzelà-Ascoli theorem for compactness.

Lemma $3.4([3])$. Let $(C(J, \mathbb{R}), \preceq,\|\cdot\|)$ be a partially ordered Banach space with the norm $\|\cdot\|$ and the order relation $\preceq$ defined by (3.10) and (3.11) respectively. Then every partially compact subset $S$ of $C(J, \mathbb{R})$ is Janhavi set.

We now state and prove our first result of the paper.

Theorem 3.3. Suppose that hypotheses $\left(H_{1}\right)-\left(H_{3}\right)$ and $(L S)$ hold. Then the BVP (1.1) and (1.2) has a solution $x^{*}$ defined on $J$ and the sequence $\left\{x_{n}\right\}_{n=0}^{\infty}$ of successive approximations defined by

$$
\begin{aligned}
& x_{0}=u \text {, } \\
& x_{n+1}(t)=z(t)+ \begin{cases}\mathcal{F}_{f} x_{n}(t), & \text { if } t \in I, \\
e^{\left(\frac{a_{j}\left(t-t_{0}\right)}{b_{j}}\right)} \mathcal{F}_{f} x_{n}\left(t_{j}\right), & \text { if } t \in I_{j}, b_{j} \neq 0, j=0,1, \\
0, & \text { if } t \in I_{j}, b_{j}=0, j=0,1,\end{cases}
\end{aligned}
$$

converges monotone nondecreasingly to $x^{*}$.

Proof. Set $E=C(J, \mathbb{R})$. Then, in view of Lemma 3.4, every compact chain $C$ in $E$ possesses the compatibility property with respect to the norm $\|\cdot\|$ and the order relation $\preceq$ so that every compact chain $C$ is a Janhavi set in $E$.

Define an operator $\mathcal{T}$ on $E$ by

$$
\mathcal{T} x(t)=z(t)+ \begin{cases}\mathcal{F}_{f} x(t), & \text { if } t \in I, \\ e^{\left(\frac{a_{j}\left(t-t_{0}\right)}{b_{j}}\right)} \mathcal{F}_{f} x\left(t_{j}\right), & \text { if } t \in I_{j}, b_{j} \neq 0, j=0,1, \\ 0, & \text { if } t \in I_{j}, b_{j}=0, j=0,1 .\end{cases}
$$

From the continuity of the integral, it follows that $\mathcal{T}$ defines the operator $\mathcal{T}: E \rightarrow$ E. Applying Lemmas 2.2 and 2.3, the BVP (1.1) and (1.2) is equivalent to the operator equation

$$
\mathcal{T} x(t)=x(t), t \in J
$$

Now, we show that the operator $\mathcal{T}$ satisfies all the conditions of Theorem 2.1 in a series of following steps.

Step I: $\mathcal{T}$ is nondecreasing on $E$. 
Let $x, y \in E$ be such that $x \succeq y$. Then, from the hypothesis $\left(\mathrm{H}_{3}\right)$ it follows that $(x, y) \rightarrow$ $f(t, x, y)$ is nondecreasing in $\mathbb{R} \times \mathbb{R}$ for almost all $t \in I$. Noticing also that $k$ is nonnegativevalued, we have that

$$
\begin{aligned}
\mathcal{F}_{f} x(t) & =\int_{t_{0}}^{t_{1}} k(t, s) f(s, x(s), x(\varphi(s))) d s \\
& \geq \int_{t_{0}}^{t_{1}} k(t, s) f(s, y(s), y(\varphi(s))) d s \\
& =\mathcal{F}_{f} y(t)
\end{aligned}
$$

for all $t \in I$. This implies that $\mathcal{F}_{f} x \succeq \mathcal{F}_{f} y$ whenever $x \succeq y$. As a result, we have that

$$
\begin{aligned}
\mathcal{T} x(t)=z(t)+ \begin{cases}\mathcal{F}_{f} x(t), & \text { if } t \in I, \\
e^{\left(\frac{a_{j}\left(t-t_{0}\right)}{b_{j}}\right)} \mathcal{F}_{f} x\left(t_{j}\right), & \text { if } t \in I_{j}, b_{j} \neq 0, j=0,1, \\
0, & \text { if } t \in I_{j}, b_{j}=0, j=0,1 .\end{cases} \\
\geq z(t)+ \begin{cases}\mathcal{F}_{f} y(t), & \text { if } t \in I, \\
e^{\left(\frac{a_{j}\left(t-t_{0}\right)}{b_{j}}\right)} \mathcal{F}_{f} y\left(t_{j}\right), & \text { if } t \in I_{j}, b_{j} \neq 0, j=0,1, \\
0, & \text { if } t \in I_{j}, b_{j}=0, j=0,1 .\end{cases} \\
=\mathcal{T} y(t),
\end{aligned}
$$

for all $t \in J$. Consequently, $\mathcal{T} x \succeq T y$ for all $x, y \in E$ with $x \succeq y$. This shows that the operator $\mathcal{T}$ is also nondecreasing on $E$.

Step II: $\mathcal{T}$ is partially continuous on $E$.

Let $\left\{x_{n}\right\}_{n \in \mathbb{N}}$ be a sequence in a chain $C$ such that $x_{n} \rightarrow x$ as $n \rightarrow \infty$. Since the $f$ is continuous, we have

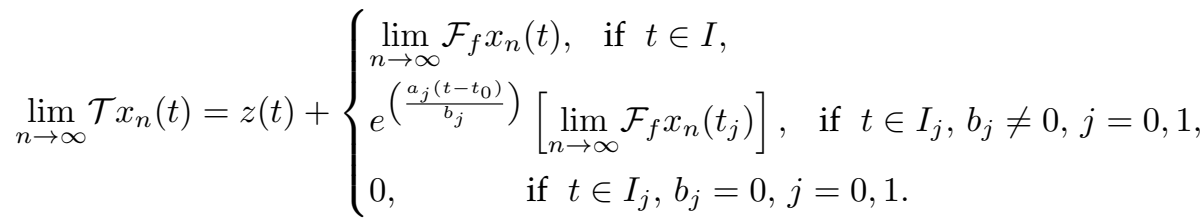

$$
\begin{aligned}
& =z(t)+ \begin{cases}\mathcal{F}_{f} x(t), & \text { if } t \in I, \\
e^{\left(\frac{a_{j}\left(t-t_{0}\right)}{b_{j}}\right)} \mathcal{F}_{f} x\left(t_{j}\right), & \text { if } t \in I_{j}, b_{j} \neq 0, j=0,1, \\
0, & \text { if } t \in I_{j}, b_{j}=0, j=0,1 .\end{cases} \\
& =\mathcal{T} x(t),
\end{aligned}
$$

for all $t \in J$. This shows that $\mathcal{T} x_{n}$ converges to $\mathcal{T} x$ pointwise on $J$.

Next, we show that $\left\{\mathcal{T} x_{n}\right\}_{n \in \mathbb{N}}$ is an equicontinuous sequence of functions in $E$. Now for any $\tau_{1}, \tau_{2} \in J$, one obtains

$$
\left|\mathcal{T} x_{n}\left(\tau_{1}\right)-\mathcal{T} x_{n}\left(\tau_{2}\right)\right| \leq\left|z\left(\tau_{1}\right)-z\left(\tau_{2}\right)\right|+\left|w\left(\tau_{1}\right)-w\left(\tau_{2}\right)\right|,
$$


where

$$
w(t)= \begin{cases}M_{f} \int_{t_{0}}^{t_{1}} k(t, s) d s, & t \in I, \\ \left(t_{1}-t_{0}\right) K M_{f} e^{\left(\frac{a_{j}\left(t-t_{0}\right)}{b_{j}}\right)}, & t \in I_{j}, b_{j} \neq 0, j=0,1, \\ 0, & t \in I_{j}, b_{j}=0, j=0,1 .\end{cases}
$$

Since the functions $t \rightarrow k(t, s)$ and $t \rightarrow e^{\left(\frac{a_{j}\left(t-t_{0}\right)}{b_{j}}\right)}$ are continuous on compact $I$ and $I_{j}$ respectively, they are uniformly continuous there. Consequently the function $t \rightarrow w(t)$ is uniformly continuous on $J$. Similarly, the function $t \rightarrow z(t)$ is also uniformly continuous on $J$. Therefore, we have

$$
\left|z\left(\tau_{1}\right)-z\left(\tau_{2}\right)\right| \rightarrow 0 \quad \text { and } \quad\left|w\left(\tau_{1}\right)-w\left(\tau_{2}\right)\right| \rightarrow 0 \quad \text { as } \tau_{1} \rightarrow \tau_{2}
$$

uniformly on $J$. As a result, we have that

$$
\left|\mathcal{T} x_{n}\left(\tau_{1}\right)-\mathcal{T} x_{n}\left(\tau_{2}\right)\right| \rightarrow 0 \quad \text { as } \tau_{1} \rightarrow \tau_{2},
$$

uniformly for all $n \in \mathbb{N}$. This shows that the convergence $\mathcal{T} x_{n} \rightarrow \mathcal{T} x$ is uniform and that $\mathcal{T}$ is a partially continuous operator on $E$ into itself.

Step III: $\mathcal{T}$ is partially compact operator on $E$.

Let $C$ be an arbitrary chain in $E$. We show that $\mathcal{B}(C)$ is uniformly bounded and equicontinuous set in $E$. First we show that $\mathcal{T}(C)$ is uniformly bounded. Let $y \in \mathcal{T}(C)$ be any element. Then there is an element $x \in C$ such that $y=\mathcal{T} x$. By hypothesis $\left(\mathrm{H}_{2}\right)$

$$
\begin{aligned}
|y(t)| \leq \begin{cases}M_{f} K\left(t_{1}-t_{0}\right), & t \in I, \\
M_{f} K\left(s_{1}-t_{0}\right) e^{\left(\frac{a_{j}\left(t-t_{0}\right)}{b_{j}}\right)}, & t \in I_{j}, b_{j} \neq 0, j=0,1, \\
0, & t \in I_{j}, b_{j}=0, j=0,1 .\end{cases} \\
\leq M_{f} K\left(s_{1}-t_{0}\right) e^{\left(\frac{a_{j}\left(s_{1}-t_{0}\right)}{b_{j}}\right)} \\
=r
\end{aligned}
$$

for all $t \in J$. Taking the supremum over $t$ we obtain $\|y\|=\|\mathcal{T} x\| \leq r$ for all $y \in \mathcal{T}(C)$. Hence $\mathcal{T}(C)$ is a uniformly bounded subset of $E$. Next we show that $\mathcal{T}(C)$ is an equicontinuous set in $E$. Let $t_{1}, t_{2} \in J$, with $t_{1}<t_{2}$. Then proceeding with the arguments that given in Step II it can be shown that

$$
\left|y\left(t_{2}\right)-y\left(t_{1}\right)\right|=\left|\mathcal{T} x\left(t_{2}\right)-\mathcal{T} x\left(t_{1}\right)\right| \rightarrow 0 \quad \text { as } \quad t_{1} \rightarrow t_{2}
$$

uniformly for all $y \in \mathcal{T}(C)$. This shows that $\mathcal{T}(C)$ is an equicontinuous subset of $E$. Now, $\mathcal{T}(C)$ is a uniformly bounded and equicontinuous subset of functions in $E$ and hence it is compact in view of Arzelá-Ascoli theorem. Consequently $\mathcal{T}: E \rightarrow E$ is a partially compact operator on $E$ into itself.

Step IV: $u$ satisfies the operator inequality $u \preceq \mathcal{T} u$.

By hypothesis (LS), the BVP (1.1) and (1.2) has a lower solution $u$ defined on $J$. Then we have

$$
\left.\begin{array}{r}
\mathcal{L} u(t) \leq f(t, u(t), u(\varphi(t))) \text { a.e. } t \in I, \\
\mathcal{B}_{j} u(t) \leq \alpha_{j}(t), t \in I_{j}, j=0,1 .
\end{array}\right\}
$$

By using this, the maximum principle [13] and the definition of $\mathcal{T}$, it can be shown that the function $u \in C(J, \mathbb{R})$ satisfies the relation $u \preceq \mathcal{T} u$ on $J$. 
Thus, $\mathcal{T}$ satisfies all the conditions of Theorem 2.1 and so the operator equation $\mathcal{T} x=x$ has a solution $x^{*}$ and the sequence $\left\{\mathcal{T}^{n} u\right\}_{n=0}^{\infty}$ of successive iterations of $\mathcal{T}$ converges monotone nondecreasingly to $x^{*}$. Therefore, the integral equation (2.5) and consequently the BVP (1.1) and (1.2) has a solution $x^{*}$ and the sequence $\left\{x_{n}\right\}_{n=0}^{\infty}$ of successive approximations defined by (3.12) converges monotone nondecreasingly to $x^{*}$. This completes the proof.

Theorem 3.4. Suppose that hypotheses $\left(H_{1}\right)-\left(H_{2}\right),\left(H_{4}\right)$ and $(L S)$ hold. Furthermore, if

$$
e^{\left(\frac{a_{j}\left(s_{1}-t_{0}\right)}{b_{j}}\right)} K\left(t_{1}-t_{0}\right) \psi_{f}(r)<r, r>0,
$$

then the BVP (1.1) and (1.2) has a unique solution $\xi^{*}$ defined on $J$ and the sequence $\left\{x_{n}\right\}_{n=0}^{\infty}$ of successive approximations defined by (3.12) converges monotone nondecreasingly to $\xi^{*}$.

Proof. Set $E=C(J, \mathbb{R})$. Define the operator $\mathcal{T}$ by (3.13). We shall show that $\mathcal{T}$ satisfies all the conditions of Theorem 2.2 in $E$. We shall simply show that the operator $\mathcal{T}$ is a partial nonlinear $\mathcal{D}$-contraction on $E$. Let $x, y \in E$ be any two elements such that $x \succeq y$. Then, by hypothesis $\left(\mathrm{H}_{3}\right)$,

$$
|\mathcal{T} x(t)-\mathcal{T} y(t)| \leq \begin{cases}\left|\mathcal{F}_{f} x(t)-\mathcal{F}_{f} y(t)\right|, & \text { if } t \in I, \\ e^{\left(\frac{a_{j}\left(t-t_{0}\right)}{b_{j}}\right)}\left|\mathcal{F}_{f} x\left(t_{j}\right)-\mathcal{F}_{f} y\left(t_{j}\right)\right|, & \text { if } t \in I_{j}, b_{j} \neq 0, j=0,1, \\ 0, & \text { if } t \in I_{j}, b_{j}=0, j=0,1 .\end{cases}
$$

Now, for any two elements $x, y \in E$ with $x \succeq y$, we have

$$
\left|\mathcal{F}_{f} x(t)-\mathcal{F}_{f} y(t)\right| \leq K\left(t_{1}-t_{0}\right) \psi_{f}(\|x-y\|)
$$

for all $t \in J$. Therefore, from the inequality (3.17) it follows that

$$
|\mathcal{T} x(t)-\mathcal{T} y(t)| \leq \begin{cases}K\left(s_{1}-t_{0}\right) \psi_{f}(\|x-y\|) \mid, & \text { if } t \in I, \\ e^{\left(\frac{a_{j}\left(s_{1}-t_{0}\right)}{b_{j}}\right)} K\left(t_{1}-t_{0}\right) \psi_{f}(\|x-y\|), & \text { if } t \in I_{j}, b_{j} \neq 0, j=0,1, \\ 0, & \text { if } t \in I_{j}, b_{j}=0, j=0,1 .\end{cases}
$$

Taking the supremum over $t$ in the above inequality (3.19), we obtain

$$
\|\mathcal{T} x-\mathcal{T} y\| \leq \psi_{\mathcal{T}}(\|x-y\|)
$$

for all $x, y \in E, x \succeq y$, where $\psi_{\mathcal{T}}(r)=e^{\left(\frac{a_{j}\left(s_{1}-t_{0}\right)}{b_{j}}\right)} K\left(t_{1}-t_{0}\right) \psi_{f}(r)<r$ for $r>0$. This shows that $\mathcal{T}$ is a partial nonlinear $\mathcal{D}$-contraction on $E$. Furthermore, it can be shown as in the proof of Theorem 3.3 that the function $u$ given in hypothesis (LS) satisfies the operator inequality $u \preceq \mathcal{T} u$ on $J$. Now a direct application of Theorem 2.2 yields that the BVP (1.1) and (1.2) has a unique solution $\xi^{*}$ defined on $J$ and the sequence $\left\{x_{n}\right\}_{n=0}^{\infty}$ of successive approximations defined by (3.12) converges monotone nondecreasingly to $\xi^{*}$.

Remark 3.1. The conclusion of Theorems 3.3 and 3.4 also remain true if we replace the hypothesis (LS) with (US). The proof of Theorems 3.3 and 3.4 under this new hypothesis is similar and can be obtained by closely observing the same arguments with appropriate modifications. In this case the sequence $\left\{x_{n}\right\}_{n=0}^{\infty}$ defined by (3.12) with $x_{0}(t)=v(t), t \in$ $[0, T]$, converges monotone nonincreasingly to the solution $x^{*}$ of he BVP (1.1) and (1.2) on $J$. 
Remark 3.2. We note that if the BVP (1.1) and (1.2) has a lower solution $u \in A C^{1}(J, \mathbb{R})$ as well as an upper solution $v \in A C^{1}(J, \mathbb{R})$ such that $u \preceq v$, then under the given conditions of Theorems 3.3 and 3.4 it has corresponding solutions $x_{*}$ and $y^{*}$ and these solutions satisfy the inequality

$$
u=x_{0} \preceq x_{1} \preceq \cdots \preceq x_{n} \preceq x_{*} \preceq y^{*} \preceq y_{n} \preceq \cdots \preceq y_{1} \preceq y_{0}=v .
$$

Hence $x_{*}$ and $y^{*}$ are respectively the minimal and maximal impulsive solutions of the HIVP (1.1) in the vector segment $[u, v]$ of the Banach space $E=C(J, \mathbb{R})$, where the vector segment $[u, v]$ is a set of elements in $C(J, \mathbb{R})$ defined by

$$
[u, v]=\{x \in C(J, \mathbb{R}) \mid u \preceq x \preceq v\} .
$$

This is because of the order cone $K$ defined by (3.11) is a closed convex subset of $C(J, \mathbb{R})$. However, we have not used any property of the cone $K$ in the main existence results of this paper. A few details concerning the order relation by the order cones and the Janhavi sets in an ordered Banach space are given in Hekkilä and Lakshmikatham [9].

Remark 3.3. Theorems 3.3 and 3.4 include the existence and approximation results for the nonlinear BVP

$$
-x^{\prime \prime}=f(t, x) \text { a.e } t \in\left[t_{0}, t_{1}\right], x\left(t_{0}\right)=0=x\left(t_{1}\right),
$$

already discussed in Dhage [3] as the special cases.

\section{THE EXAMPLES}

Example 4.1. Choose $I=[-1,1]$ and $J=[-2,2]$. Consider the nonlinear BVP

$$
\left.\begin{array}{rl}
-x^{\prime \prime}(t) & =\tanh x(t)+\tanh x(t-1)+\tanh x(t+1) \text { a.e. } t \in I, \\
x(t) & =1, t \in[-2,-1] \cup[1,2] .
\end{array}\right\}
$$

Here, $f(t, x, y, z)=\tanh x+\tanh y+\tanh z$ for $t \in[-1,1]$ and $x, y, z \in \mathbb{R}$. Then $f$ is continuous and bounded on $I \times \mathbb{R} \times \mathbb{R} \times \mathbb{R}$ with bound $M_{f}=3$. Also $f(t, x, y, z)$ is nondecreasing in the variables $x, y$ and $z$ for each $t \in I$.

Now the BVP (4.21) is equivalent to the nonlinear integral equation

$$
x(t)=1+\left\{\begin{array}{l}
\int_{-1}^{1} k(t, s)[\tanh x(s)+\tanh x(s-1)+\tanh x(s+1)] d s, t \in I, \\
0, \quad t \in[-2,-1] \cup[1,2],
\end{array}\right.
$$

where $k$ is a Green's function associated with the homogeneous BVP

$$
-x^{\prime \prime}(t)=0, t \in[-1,1], \quad x(-1)=0=x(1),
$$

defined by

$$
k(t, s)= \begin{cases}\frac{(1-t)(1+s)}{2}, & -1 \leq s \leq t \leq 1, \\ \frac{(1+t)(1-s)}{2}, & -1 \leq t \leq s \leq 1,\end{cases}
$$

which is continuous and nonnegative on $I \times I$.

It can be verified that the function $u \in C(J, \mathbb{R})$ defined by

$$
u(t)=1+\left\{\begin{array}{l}
-3 \int_{-1}^{1} k(t, s) d s, \quad t \in[-1,1] \\
0, \quad t \in[-2,-1] \cup[1,2]
\end{array}\right.
$$


is a lower solution of the BVP (4.21) on $J$. Similarly, the function $v \in C(J, \mathbb{R})$ defined by

$$
v(t)=1+\left\{\begin{array}{l}
3 \int_{-1}^{1} k(t, s) d s, \quad t \in[-1,1], \\
0, \quad t \in[-2,-1] \cup[1,2],
\end{array}\right.
$$

is an upper solution of the BVP (4.21) on $J$.

Hence, by an application of Theorem 3.3, the BVP (4.21) has a solution $x^{*}$ and the sequence $\left\{x_{n}\right\}_{n=0}^{\infty}$ of successive approximations defined by

$$
\begin{aligned}
& x_{0}(t)=1+\left\{\begin{array}{l}
-3 \int_{-1}^{1} k(t, s) d s, t \in[-1,1], \\
0, \quad t \in[-2,-1] \cup[1,2],
\end{array}\right. \\
& x_{n+1}(t)=1+\left\{\begin{array}{l}
\int_{-1}^{1} k(t, s)\left[\tanh x_{n}(s)+\tanh x_{n}(s-1)+\tanh x_{n}(s+1)\right] d s, t \in I, \\
0, \quad t \in[-2,-1] \cup[1,2],
\end{array}\right.
\end{aligned}
$$

converges monotone nondecreasingly to $x^{*}$. Similarly, the sequence $\left\{y_{n}\right\}_{n=0}^{\infty}$ of successive approximations defined by

$$
\begin{aligned}
& y_{0}(t)=1+\left\{\begin{array}{l}
3 \int_{-1}^{1} k(t, s) d s, \quad t \in[-1,1], \\
0, \quad t \in[-2,-1] \cup[1,2]
\end{array}\right. \\
& y_{n+1}(t)=1+\left\{\begin{array}{l}
\int_{-1}^{1} k(t, s)\left[\tanh y_{n}(s)+\tanh y_{n}(s-1)+\tanh y_{n}(s+1)\right] d s, t \in I, \\
0, \quad t \in[-2,-1] \cup[1,2],
\end{array}\right.
\end{aligned}
$$

converges monotone nonincreasingly to the solution $y^{*}$. Furthermore, we have

$$
\begin{aligned}
u(t) & =1+\left\{\begin{array}{l}
-3 \int_{-1}^{1} k(t, s) d s, \quad t \in[-1,1], \\
0, \quad t \in[-2,-1] \cup[1,2],
\end{array}\right. \\
& \leq 1+\left\{\begin{array}{l}
3 \int_{-1}^{1} k(t, s) d s, \quad t \in[-1,1], \\
0, \quad t \in[-2,-1] \cup[1,2],
\end{array}\right. \\
& =v(t)
\end{aligned}
$$

for all $t \in J$ and so $u \preceq v$. Therefore, in view of Remark 3.2, $x^{*}$ and $y^{*}$ are respectively the minimal and maximal solutions of the BVP (4.21) on $J$ in the vector segment $[u, v]$ of the ordered Banach space $C(J, \mathbb{R})$ with

$$
u=x_{0} \preceq x_{1} \preceq \cdots \preceq x_{n} \preceq x^{*} \preceq y^{*} \preceq y_{n} \preceq \cdots \preceq y_{1} \preceq y_{0}=v .
$$

Example 4.2. Given closed and bounded intervals $I=[-1,1]$ and $J=[-2,2]$, consider the nonlinear boundary value problem

$$
\left.\begin{array}{rl}
-x^{\prime \prime}(t) & =\frac{2}{3}\left[\tan ^{-1} x(t)+\tan ^{-1} x(t-1)+\tan ^{-1} x(t+1)\right] \text { a.e. } t \in I, \\
x(t) & =1, t \in[-2,-1] \cup[1,2] .
\end{array}\right\}
$$

Here, $f(t, x, y, z)=\frac{2}{3}\left[\tan ^{-1} x+\tan ^{-1} y+\tan ^{-1} z\right]$ for $t \in[-1,1]$ and $x, y, z \in \mathbb{R}$. Then $f$ is continuous and bounded on $I \times \mathbb{R} \times \mathbb{R} \times \mathbb{R}$ with bound $M_{f}=1$. Also $f(t, x, y, z)$ is nondecreasing in the variables $x, y$ and $z$ for each $t \in I$. Moreover, $f$ satisfies the hypothesis $\left(H_{4}\right)$ 
with $\psi_{f}(r)=\frac{r}{1+\xi}$ for some $0 \leq \xi \leq r$ and consequently, the condition (3.17) of Theorem 3.4 is satisfied. Now the BVP (4.25) is equivalent to the nonlinear integral equation

$$
x(t)=1+\left\{\begin{array}{l}
\frac{2}{3} \int_{-1}^{1} k(t, s)\left[\tan ^{-1} x(s)+\tan ^{-1} x(s-1)+\tan ^{-1} x(s+1)\right] d s, t \in I, \\
0, \quad t \in[-2,-1] \cup[1,2],
\end{array}\right.
$$

where $k$ is a Green's function given by (4.24).

It can be verified that the function $u \in C(J, \mathbb{R})$ defined by

$$
u(t)=1+\left\{\begin{array}{l}
-4 \int_{-1}^{1} k(t, s) d s, \quad t \in[-1,1] \\
0, \quad t \in[-2,-1] \cup[1,2]
\end{array}\right.
$$

is a lower solution of the BVP (4.25) on $J$. Similarly, the function $v \in C(J, \mathbb{R})$ defined by

$$
v(t)=1+\left\{\begin{array}{l}
4 \int_{-1}^{1} k(t, s) d s, \quad t \in[-1,1], \\
0, \quad t \in[-2,-1] \cup[1,2]
\end{array}\right.
$$

is an upper solution of the BVP (4.25) on J.

Hence, by an application of Theorem 3.4, the BVP (4.25) has a unique solution $\xi^{*}$ and the sequence $\left\{x_{n}\right\}_{n=0}^{\infty}$ of successive approximations defined by

$$
\begin{aligned}
x_{0}(t)=1+\left\{\begin{array}{l}
-4 \int_{-1}^{1} k(t, s) d s, \quad t \in[-1,1], \\
0, \quad t \in[-2,-1] \cup[1,2],
\end{array}\right. \\
x_{n+1}(t)=1+\left\{\begin{array}{l}
\frac{2}{3} \int_{-1}^{1} k(t, s)\left[\tan ^{-1} x_{n}(s)+\tan ^{-1} x_{n}(s-1)+\tan ^{-1} x_{n}(s+1)\right] d s, t \in I, \\
0, \quad t \in[-2,-1] \cup[1,2],
\end{array}\right.
\end{aligned}
$$

converges monotone nondecreasingly to $\xi^{*}$. Similarly, the sequence $\left\{y_{n}\right\}_{n=0}^{\infty}$ of successive approximations defined by

$$
\begin{aligned}
y_{0}(t)=1+\left\{\begin{array}{l}
4 \int_{-1}^{1} k(t, s) d s, \quad t \in[-1,1], \\
0, \quad t \in[-2,-1] \cup[1,2],
\end{array}\right. \\
y_{n+1}(t)=1+\left\{\begin{array}{l}
\frac{2}{3} \int_{-1}^{1} k(t, s)\left[\tan ^{-1} y_{n}(s)+\tan ^{-1} y_{n}(s-1)+\tan ^{-1} y_{n}(s+1)\right] d s, t \in I, \\
0, \quad t \in[-2,-1] \cup[1,2],
\end{array}\right.
\end{aligned}
$$

converges monotone nonincreasingly to the unique solution $\xi^{*}$. Furthermore, since $u \preceq v$, the functions $\xi^{*}$ is a unique solution of the BVP (4.25) in the vector segment $[u, v]$ with the relation

$$
u=x_{0} \preceq x_{1} \preceq \cdots \preceq x_{n} \preceq \xi^{*} \preceq y_{n} \preceq \cdots \preceq y_{1} \preceq y_{0}=v .
$$

\section{THE CONCLUSION}

Finally in the conclusion, we mention that the existence and approximation results for the BVP (1.1) and (1.2) on $J$ may also be obtained by using other iteration methods already known in the literature. In case of well-known Picard iteration method, the nonlinearity $f$ is required to satisfy a certain so called strong Lipschitz condition whereas in our Theorems 3.3 and 3.4, it is not a requirement. Similarly, in the case of monotone iterative technique for the BVP (1.1) and (1.2), we need to have the existence of both comparable lower as well as upper solutions along with a cumbersome comparison result for getting theoretic approximation of the solution (see $[11,12]$ and references therein). However, here 
in the present approach of this paper, we get rid of above stringent conditions and still obtain the existence of an approximate solution in an easy straight forward way. Again, in the case of existence result via generalized iteration method developed by Heikkilä and Lakshmikantham [9] (see also Dhage and Heikkilä [8] and references therein), we also need the existence of both comparable upper as well as lower solutions together with some other conditions such as integrability of the nonlinearity $f$, notwithstanding it does not yield any algorithm for the solution. Furthermore, the conclusion of the upper and lower solutions method is a by-product of our monotone iteration method as mentioned in Remark 3.2. Therefore, in view of the above observations, we conclude that our monotone iteration method of this paper is an elegant, relatively better and more powerful than all the above mentioned frequently used iteration methods because it provides the additional information of the algorithm along with the monotonic characterization of the convergence of the sequence of iterations to the approximate solution of the BVP (1.1) and (1.2) defined on $J$ under weaker conditions.

Acknowledgment. The authors are thankful to the referees for pointing out typos and giving some suggestions for the improvement of the paper.

\section{REFERENCES}

[1] Dhage, B.C. Partially condensing mappings in partially ordered normed linear spaces and applications to functional integral equations. Tamkang J. Math. 45 (2014), no. 4, 397-427.

[2] Dhage, B. C. Some generalizations of a hybrid fixed point theorem in a partially ordered metric space and nonlinear functional integral equations. Differ. Equ. Appl. 8 (2016), 77-97.

[3] Dhage, B. C. Approximation and existence of solutions for nonlinear two point BVPs of ordinary second order differential equations. Nonlinear Studies 23 (2016), no. 1, 1-17.

[4] Dhage, B. C. Coupled and mixed coupled hybrid fixed point principles in a partially ordered Banach algebra and PBVPs of nonlinear coupled quadratic differential equations. Differ. Equ. Appl. 11 (2019), no. 1, $1-85$.

[5] Dhage, B. C.; Dhage, S. B. Hybrid fixed point theory for nonincreasing mappings in partially ordered metric spaces and applications. J. Nonlinear Anal. Appl. 5 (2014), no. 2, 71-79.

[6] Dhage, B. C.; Dhage, S. B. Approximating solutions of nonlinear PBVPs of hybrid differential equations via hybrid fixed point theory. Indian J. Math. 57 (2015), 103-119.

[7] Dhage, S. B.; Dhage, B. C.; Graef, J. B. Dhage iteration method for initial value problem for nonlinear first order integrodifferential equations. J. Fixed Point Theory Appl. 18 (2016), 309-325.

[8] Dhage, B. C.; Heikkilä, S. On nonlinear boundary value problems with deviating arguments and discontinuous right hand side. J. Appl. Math. Stoch. Anal. 6 (1993), no. 1, 83-92

[9] Heikkilä, S.; Lakshmikantham, V. Monotone iterative techniques for discontinuous nonlinear differential equations. Marcel Dekker inc., New York 1994.

[10] Heikkilä, S.; Mooney, J. W. Seikkala, S. Existence, uniqueness and comparison results for nonlinear boundary value problems involving a deviating argument. J. Differ. Equa. 41 (1981), no. 3, 320-333.

[11] Kamaljeet, Bahuguna, D. Monotone iterative technique for nonlocal fractional differential equations with finite delay in Banach space. Electronic J. Qualitative Theory Diff. Equa. 9 (2015), 1-16.

[12] Ladde, G. S.; Lakshmikantham, V.; Vatsala, A. S. Monotone iterative techniques for nonlinear differential equations. Vol. 27, Pitman 1985.

[13] Protter, M. H.; Weinberger, H. F. Maximum principles in differential equations. Prentice-Hall, Englewood Cliffs, N. J., 1967.

\footnotetext{
1 "KASUBAI", GURUKUl COLONY

THODGA ROAD
}

AHMEDPUR 413515, DisT. LATUR, (M.S.) INDIA

Email address: bcdhage@gmail.com, jbdhage@gmail.com

${ }^{2}$ Aligarh Muslim UNIVERSITy

DEPARTMENT OF MATHEMATICS

ALIGARH 202002, INDIA

Email address: javid.mmeamu.ac.in 